\title{
Biological activity of the new photoactive ruthenium nitrosyl complexes: cytotoxicity and effects on DNA repair
}

\author{
D.V. Khantakova ${ }^{1,2}$, D.V. Petrova ${ }^{1,2}$, I.R. Grin ${ }^{1,2 *}$ \\ ${ }^{1}$ Institute of Chemical Biology and Fundamental Medicine SB RAS, Novosibirsk, Russia \\ ${ }^{2}$ Novosibirsk State University, Novosibirsk, Russia \\ *e-mail:grin@niboch.nsc.ru
}

Key words: photopharmacology, MTT assay, ruthenium nitrosyl complex, nitric oxide, DNA repair, BER

Motivation and Aim: Nitric oxide plays an important role in different biological processes in healthy cells, such as neurotransmission, blood pressure control, immunity, and also in the process of protecting the body against the spread of cancer cells during metastasis. The biological activity of ruthenium nitrosyl complexes is mainly related to the possibility of NO release in biological solutions due to photochemical activation or reduction by biological reductants such as ascorbic acid or NADH. On the other hand, it is known that a solution saturated with NO gas is capable of inhibiting the activity of the prokaryotic DNA glycosylase Nth, the main player of the base excision DNA repair (BER). Thus, the study of both the antiproliferative potential of nitrosyl ruthenium complexes on human cell lines, such as the study of the activity of the eukaryotic DNA repair enzymes under conditions of NO saturation in vitro, is necessary for understanding the mechanisms of inhibiting the proliferation of cancer cells.

Methods and Algorithms: We measured the cytotoxicity potential in human cell lines of the recently synthesized ruthenium nitrosyl complexes lines by MMT analysis. To analyzed in vitro activity of the eukaryotic DNA repair enzymes we used NO saturation conditions controlled by photochemical activation.

Results: In recent decades, a new direction inmedicine has developed-photopharmacology, in particular, ruthenium nitrosyl complexes provide the possibility for the photodynamic therapy as potential anticancer drug. Measuring of the cytotoxicity the ruthenium nitrosyl complexes in human mammary adenocarcinoma (MCF-7) and immortalized human embryonic kidney (HEK-293) cell lines revealed that the $\left[\mathrm{RuNO}(\text { betta - Pic })_{2}\left(\mathrm{NO}_{2}\right)_{2} \mathrm{OH}\right]$ complex exhibited inhibition of the growth of cancer cells at remarkably low micromolar concentrations. This ruthenium nitrosyl complex inhibited the activity some eukaryotic BER enzymes containing [4Fe-4S] cluster after controlled photoinduction in vitro. Thus, the ruthenium nitrosyl complexes seem are very promising for photocontrolled NOdependent toxicity conditions for in vitro applications and research.

Acknowledgements: Supported under SB RAS Integrated scientific program, No. II.1/16 (0309-2018-0008). 